\title{
A new hierarchical classification of causes of infant deaths in England and Wales
}

\author{
Eva Alberman, Beverley Botting, Nick Blatchley, Alan Twidell
}

Department of

Environmental and

Preventive Medicine,

Wolfson Institute of

Preventive Medicine,

Medical College of St

Bartholomew's

Hospital, London

E Alberman

Health Statistics,

Office of Population

Censuses and Surveys,

London

B Botting

N Blatchley

Alan Twidell

Correspondence to: Professor Eva Alberman

Department of

Environmental and

Environmental and
Preventive Medicine

Preventive Medicine,
Wolfson Institute of

Preventive Medicine,

Medical College of St

Bartholomew's Hospital,

Charterhouse Square,

London EC1M 6BQ.

Accepted 7 February 1994

\begin{abstract}
In 1986 The Office of Population Censuses and Surveys (OPCS) introduced new certificates for stillbirths and neonatal deaths. This allowed certifiers more flexibility in the completion of the certificate, and the number and ordering of the causes given. Tabulations have been published of the fetal and maternal causes of death mentioned on the certificates for every year from 1986 to 1991 in annual reference volumes. It has not been possible either to derive a single cause group for each death, however, or to compare the information available on neonatal deaths with that on postneonatal deaths, which are still derived from the standard death certificate. The aim of the work described here was to adapt previous classifications to derive a single cause grouping for stillbirths and infant deaths which would provide the maximum information about preventability and yet meet the national and international responsibilities of OPCS. The methods used and the tests carried out on the validity and consistency of the chosen classification are described. (Arch Dis Child 1994; 70: 403-409)
\end{abstract}

It has long been acknowledged that an analysis of causes of perinatal deaths is not complete without taking into account maternal as well as fetal factors. ${ }^{1-3}$ Baird and Thomson, ${ }^{4}$ however, also acknowledged that 'is not easy to classify

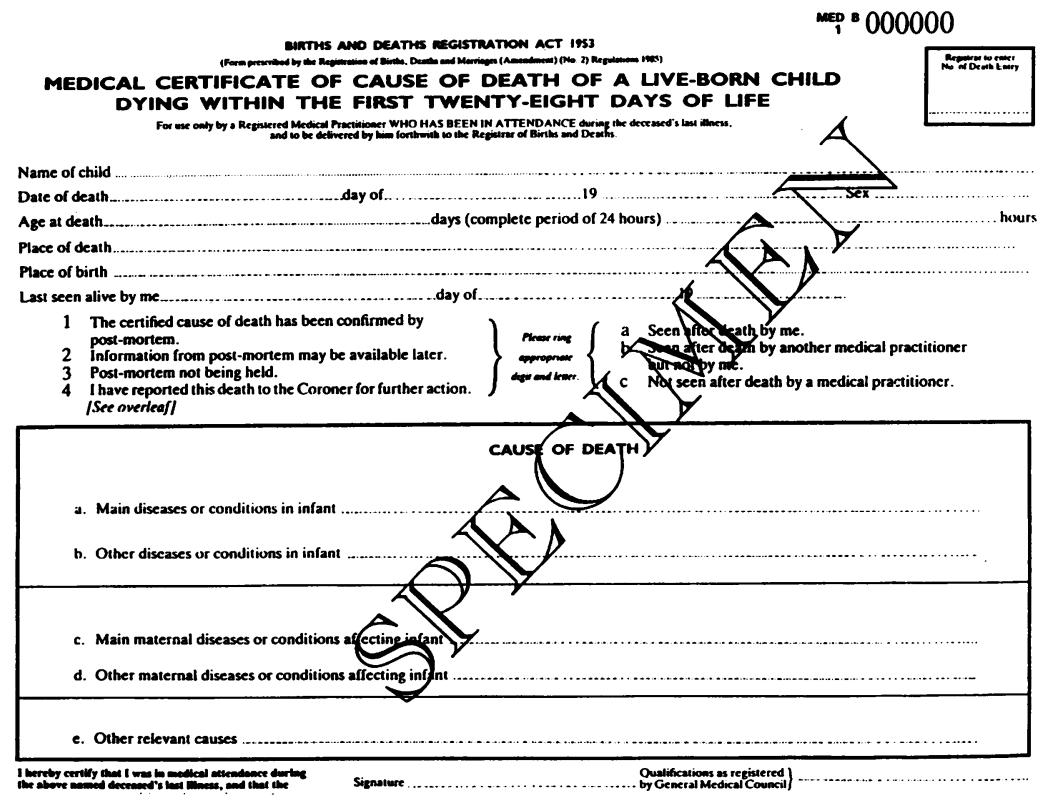

Figure 1 Medical certificate of cause of death of a live born child dying within the first 28 days of life. the causes of perinatal deaths in an informative way ... An unduly complex classification may create difficulties during statistical analysis and will tend to obscure broad trends. We consider it is desirable wherever possible to allocate each case to a single cause group, which gives the maximum information with regard to preventability'. It is this philosophy which underlies the more recent attempts by clinicians to classify early deaths. ${ }^{5-8}$

Such classifications could not be applied to the causes of perinatal deaths derived from death certificates in England and Wales before 1986 because causes of neonatal deaths were derived from the standard medical certificate used for deaths of all ages, and differed from those derived from stillbirth certificates which asked for information on maternal and fetal causes. Moreover, the format of the two systems required the medical certifier to select a single underlying cause, the choice of which would not necessarily be compatible with the philosophy described above. In 1986, in response to national and World Health Organisation recommendations for the introduction of a certificate which would elicit similar data for stillbirths and neonatal deaths, ${ }^{9} 10$ the Office of Population Censuses and Surveys (OPCS) introduced revised certificates for stillbirths and deaths of live births occurring up to 27 completed days. This allowed certifiers more flexibility in the completion of the certificate, and the number and ordering of the causes given. Because of the small number of postneonatal deaths other than congenital anomalies attributable to maternal or perinatal causes, the use of the new certificate was not extended to postneonatal deaths. ${ }^{11}$

The background to this change and the results of a pilot investigation of the use of the new forms were published in $1985,{ }^{12}$ but the format was subsequently altered to allow more than one main fetal and maternal cause to be given (fig 1). The line asking for 'other relevant causes' has been reserved for codes for external causes of violence or accident, or for the type of medical interventions, and any other causes given on this line are coded as for the 'other' causes.

The OPCS has published tabulations of the fetal and maternal causes mentioned on the certificates for every year from 1986 to 1991 in the annual reference volumes for childhood mortality ${ }^{13}$ and for perinatal and infant mortality. ${ }^{14} \mathrm{Up}$ to the present time, however, it has not been possible either to derive a single cause group for each death, or to compare the new information available on neonatal with that on postneonatal deaths. Causes of 
postneonatal deaths are still derived from the standard death certificate with a single underlying cause extracted as in the past.

There was clearly a need for a classification system which would allow the derivation in a systematic way of a single cause group, compatible across stillbirths, neonatal, and postneonatal deaths. There are, however, special characteristics needed for a classification introduced for presenting national statistics. ${ }^{15}$ This needs to be robust in spite of the lack of clinical details; to be compatible with existing regional, national, and international classifications; to be capable of giving consistent year-on-year trends; and for its introduction not to lead to abrupt changes in previous time trends. Cole et al described an international classification for infant deaths which met many of these needs. ${ }^{15}$ This was adapted from the growing amount of published work on classifications of perinatal deaths, largely produced by clinicians who had available clinical information additional to that entered on the death certificate.

None of these could be applied directly to the multicause type of data now collected for stillbirths and neonatal deaths in England and Wales, however, for which no existing classification has been found to be adequate. The aim of the work described in this paper was to adapt previous classifications to derive a single cause grouping for stillbirths and infant deaths which would 'provide maximum information regarding preventability' and yet meet the national and international responsibilities of OPCS. This work is described, as are the methods used to test the validity and consistency of the chosen classification. This account applies to infant deaths only. A subsequent paper will present the results of applying the classification to stillbirths.

\section{Methods}

\section{RATIONALE OF THE GROUPING}

The aim was to exploit the range of cause of death information available to identify a single cause which was the first in a hierarchy of events to lead to the neonatal death. The method used to identify these was established with the help of an expert group, which included paediatric pathologists, paediatricians, obstetricians, and specialists in public health. As a preliminary, infant and maternal conditions for neonatal deaths and the underlying cause of postneonatal deaths were grouped according to the main stage in the development of the fetus or infant at which they probably first acted, thus providing a basis for planning preventative action. The final grouping consisted of nine, mutually exclusive, categories:

Before the onset of labour

1 Congenital defects

2 Antepartum infections

3 Conditions related to immaturity

In or shortly after labour

4 Asphyxia, anoxia, or trauma

\section{Postnatal}

5 External conditions

6 Infections

7 Other specific conditions

9 Sudden infant deaths

Unclassified

0 Other conditions

Code 8 is used only for a category among stillborn infants.

\section{METHODS USED FOR THE ALLOCATION OF THE CLASSIFICATION}

For neonates, draft algorithms were prepared which used all mentions on a certificate, after coding using the ninth revision of the International Classification of Diseases (ICD), to allocate each death to one of the described groups. A major feature was the splitting off at the outset of the stillbirths, which subsequently followed a path separate from, but related to, that of deaths of live births. Where there was more than one cause mentioned on the certificate, the determinant of the class to which the case was allocated was that cause which allocated the death to the earliest of the possible groups in the hierarchy. This included the investigation of using 'other infant' or 'other maternal' mentions to be chosen as the determining factor if they directed the death to an earlier group in the list than would mentions given as 'main infant' or 'main maternal' conditions.

Computer programs for three versions of the algorithm were written and tested on sample data for the Northern Regional Health Authority and one other region for one year. The first version was based on any mention on any line, except on that for other relevant causes (which are used for special codes for accidents and violence). The second algorithm was based only on the first mention on the main infant line and the third on all main infant and all main maternal mentions, but excluded consideration of other mentions. Eighty eight per cent of neonatal deaths in the sample data retained the same classification for all three of these bases. This being so, it was decided to use the first algorithm to use the data to the maximum.

Dr Hey of the Northern Regional Health Authority then carried out an additional check on the grouping of individual cases for the sample for which he had additional clinical data obtained in the course of producing the regional health authority's annual report on perinatal and late neonatal deaths. As a consequence, minor changes were made to the algorithm, leading to one which was close to, but not identical with that used in the Northern region. The main differences are that: (a) the OPCS algorithm does not, in the first instance, use birth weight (which is not available until the death certificate is linked with the corresponding birth certificate); (b) that because of the importance of deaths certified as due to antepartum infection the OPCS Medical Advisory Committee requested that these were selected before those classed as related to 
Table 1 Range of International Classification of Diseases (ICD) codes used in different categories in the algorithm

\begin{tabular}{|c|c|c|c|c|c|c|c|}
\hline \multicolumn{2}{|c|}{ Category } & \multicolumn{2}{|c|}{$\begin{array}{l}\text { Range of } \\
\text { ICD codes }\end{array}$} & \multicolumn{2}{|c|}{ Category } & \multicolumn{2}{|c|}{$\begin{array}{l}\text { Range of } \\
\text { ICD codes }\end{array}$} \\
\hline 1 & Congenital malformations or defects & & & 6 & Infections & & \\
\hline & Defects of amino acid metabolism & $270 \cdot 0$ & 273.9 & & Infections and parasitic disease excluding & $001 \cdot 0$ & $088 \cdot 0$ \\
\hline & Other and unspecified metabolic disorders & $277 \cdot 0$ & $277 \cdot 9$ & & congenital syphilis & $091 \cdot 0$ & $139 \cdot 0$ \\
\hline & Disorders of immune mechanism (not AIDS) & $\left\{\begin{array}{l}279 \cdot 0 \\
279 \cdot 2\end{array}\right.$ & $\begin{array}{l}279 \cdot 0 \\
279 \cdot 9\end{array}$ & & $\begin{array}{l}\text { Abscess of thymus gland } \\
\text { Meningitis/intracranial abscess }\end{array}$ & $\begin{array}{r}254 \cdot 1 \\
320 \cdot 0\end{array}$ & $254 \cdot 1$ \\
\hline & Hereditary haemolytic anaemia & $282 \cdot 0$ & $\begin{array}{l}21 \cdot 9 \cdot 9 \\
282 \cdot 9\end{array}$ & & $\begin{array}{l}\text { Otitis media } \\
\text { Otracranial abscess }\end{array}$ & $\begin{array}{l}320 \cdot 0 \\
382 \cdot 0\end{array}$ & $\begin{array}{l}320 \cdot 0 \\
382 \cdot 9\end{array}$ \\
\hline & Constitutional aplastic anaemia & $284 \cdot 0$ & $284 \cdot 0$ & & Acute cardiac infections & $420 \cdot 0$ & $422 \cdot 9$ \\
\hline & Coagulation defects & $286 \cdot 0$ & $286 \cdot 4$ & & Acute upper respiratory infections & $460 \cdot 0$ & $466 \cdot 1$ \\
\hline & Primary thrombocytopenia & $287 \cdot 3$ & $287 \cdot 3$ & & Peritonsillar or laryngeal infections & $475 \cdot 0$ & $476 \cdot 1$ \\
\hline & Disorders of neutrophils & $288 \cdot 1$ & $288 \cdot 2$ & & Pneumonia, influenza, bronchitis & $480 \cdot 0$ & $491 \cdot 9$ \\
\hline & Cerebral degenerations & $330 \cdot 0$ & $330 \cdot 9$ & & Bronchiectasis & $494 \cdot 0$ & $494 \cdot 0$ \\
\hline & Werdnig-Hoffman disease & $335 \cdot 0$ & $335 \cdot 0$ & & Empyema, pleurisy & $510 \cdot 0$ & $511 \cdot 9$ \\
\hline & Infantile cerebral palsy & $343 \cdot 0$ & $343 \cdot 9$ & & Abscess of lung or mediastinum & $513 \cdot 0$ & $513 \cdot 1$ \\
\hline & Cerebral cysts & $348 \cdot 0$ & $348 \cdot 0$ & & Appendicitis & $540 \cdot 0$ & $542 \cdot 0$ \\
\hline & Muscular dystrophy/myopathy & $359 \cdot 0$ & $359 \cdot 3$ & & Peritonitis & $567 \cdot 0$ & $567 \cdot 9$ \\
\hline & & $424 \cdot 0$ & $424 \cdot 3$ & & Infections of kidney. & $590 \cdot 0$ & $590 \cdot 9$ \\
\hline & Endomyocardial fibrosis etc & $\{425 \cdot 0$ & $425 \cdot 1$ & & Other urinary tract infection & $\begin{array}{l}599 \cdot 0 \\
771 \cdot 3\end{array}$ & $\begin{array}{l}599 \cdot 0 \\
771 \cdot 8\end{array}$ \\
\hline & Conduction disorders & $1425 \cdot 3$ & $425 \cdot 4$ & & $\begin{array}{l}\text { Other specific neonatal infections } \\
\text { Other specific conditions }\end{array}$ & $771 \cdot 3$ & $771 \cdot 8$ \\
\hline & & $426 \cdot 0$ & $426 \cdot 9$ & $T$ & $\begin{array}{l}\text { Other specific conditions } \\
\text { Malignant or endocrine disorder }\end{array}$ & & \\
\hline & & $571 \cdot 4$ & $571 \cdot 9$ & & $\begin{array}{l}\text { Malignant or endocrine disorder } \\
\text { Thymus disorder excluding abscess }\end{array}$ & $140 \cdot 0$ & 253.9 \\
\hline & $\begin{array}{l}\text { Chromosomal abnormality of fetus ( } \\
\text { Congenital malformation (of) }\end{array}$ & $655 \cdot 1$ & $655 \cdot 1$ & & $\begin{array}{l}\text { nus disorder excluding abscess } \\
\text { nal disorder }\end{array}$ & $254 \cdot 2$ & $254 \cdot 9$ \\
\hline & & & & & $\begin{array}{l}\text { Adrenal disorder } \\
\text { Acquired haemolytic }\end{array}$ & $255 \cdot 0$ & $255 \cdot 9$ \\
\hline & $\begin{array}{l}\text { Central nervous system } \\
\text { Ear, face, and neck }\end{array}$ & $\begin{array}{l}740 \cdot 0 \\
744 \cdot 0\end{array}$ & $\begin{array}{l}743 \cdot 9 \\
744 \cdot 9\end{array}$ & & $\begin{array}{l}\text { Acquired haemolytic anaemia } \\
\text { Acquired coagulation deficiency (excluding neonatal }\end{array}$ & $283 \cdot 0$ & 283.9 \\
\hline & Heart & $\{745 \cdot 0$ & $746 \cdot 9$ & & vitamin $\mathrm{K}$ deficiency & $286 \cdot 7$ & $286 \cdot 7$ \\
\hline & Heart & $747 \cdot 1$ & $747 \cdot 9$ & & Maternal cerebral degene & $331 \cdot 0$ & $331 \cdot 9$ \\
\hline & Respiratory system & $748 \cdot 0$ & $748 \cdot 9$ & & pericardium & $423 \cdot 0$ & $423 \cdot 9$ \\
\hline & Cleft palate/cleft lip & $749 \cdot 0$ & $749 \cdot 2$ & & Cardiac dysrhythmia & $427 \cdot 0$ & $427 \cdot 9$ \\
\hline & Other of upper alimentary tract & $750 \cdot 1$ & 751.9 & & Aneurysm & $441 \cdot 0$ & $442 \cdot 9$ \\
\hline & Anomalies of female genitalia & $752 \cdot 4$ & $752 \cdot 4$ & & Asthma or bronchiectasis & $493 \cdot 0$ & $494 \cdot 0$ \\
\hline & Anomalies of male genitalia & $752 \cdot 6$ & $752 \cdot 9$ & & Non-infective enteritis/colitis & $556 \cdot 0$ & $558 \cdot 9$ \\
\hline & Anomalies of the urinary system & $753 \cdot 0$ & $753 \cdot 9$ & & Maternal infection & $760 \cdot 2$ & $760 \cdot 2$ \\
\hline & Certain other musculoskeletal & $\left\{\begin{array}{l}754 \cdot 3 \\
754 \cdot 8\end{array}\right.$ & $754 \cdot 3$ & & Maternal injury or surgery & $\begin{array}{l}760 \cdot 5 \\
760.3\end{array}$ & $\begin{array}{l}760 \cdot 6 \\
762 \cdot 3\end{array}$ \\
\hline & & $\begin{aligned} 754 \cdot 8 \\
755 \cdot 2\end{aligned}$ & $\begin{array}{l}754 \cdot 8 \\
757 \cdot 3\end{array}$ & & Other anomalies of chorion or amnion & $\begin{array}{l}102 \cdot 3 \\
762 \cdot 8\end{array}$ & $\begin{array}{l}102 \cdot 3 \\
762 \cdot 9\end{array}$ \\
\hline & And integument & $\{757 \cdot 8$ & $757 \cdot 9$ & & Fetal blood loss from cord or placenta & $772 \cdot 0$ & $772 \cdot 0$ \\
\hline & Chromosomal anomalies & $758 \cdot 0$ & $758 \cdot 9$ & & Other fetal blood loss & $772 \cdot 3$ & $772 \cdot 9$ \\
\hline & ecified anomalies & $759 \cdot 0$ & $759 \cdot 9$ & & Isoimmunisation & $773 \cdot 0$ & $773 \cdot 5$ \\
\hline & Perinatal jaundice due to hereditary anaemia & $774 \cdot 0$ & $774 \cdot 0$ & & Perinatal jaundice - other excessive haemolysis & $774 \cdot 1$ & $774 \cdot 1$ \\
\hline & Meconium ileus due to mucoviscidosis & $777 \cdot 0$ & $777 \cdot 0$ & & Perinatal jaundice remaining causes & $774 \cdot 3$ & $774 \cdot 6$ \\
\hline 2 & Antepartum infections & & & & Neonatal endocrine/metabolic disturbance & $775 \cdot 0$ & $775 \cdot 9$ \\
\hline & Congenital syphilis & $090 \cdot 0$ & $090 \cdot 9$ & & Fetal/neonatal haematological disorders & $\left\{\begin{array}{l}776 \cdot 0 \\
776 \cdot 7\end{array}\right.$ & $776 \cdot 5$ \\
\hline & Congenital AIDS & $279 \cdot 1$ & $279 \cdot 1$ & & & +1 & $776 \cdot 9$ \\
\hline & Maternal virus disease damaging fetus & $655 \cdot 3$ & $655 \cdot 3$ & & Perinatal disorders of digestive system & $\{777 \cdot 1$ & $777 \cdot 4$ \\
\hline & Chorioamnionitis & $762 \cdot 7$ & $762 \cdot 7$ & & & $1777 \cdot 6$ & $777 \cdot 9$ \\
\hline & Congenital pneumonia & $770 \cdot 0$ & $770 \cdot 0$ & & Hydrops fetalis not due to isoimmunisation & $778 \cdot 0$ & $778 \cdot 0$ \\
\hline & Other congenital infections & $771 \cdot 0$ & $771 \cdot 2$ & & Neonatal temperature regulation problems & $778 \cdot 4$ & $778 \cdot 9$ \\
\hline 3 & Immaturity & & & & Neonatal drug reactions/intoxications & $779 \cdot 4$ & $779 \cdot 5$ \\
\hline & & $761 \cdot 0$ & $761 \cdot 1$ & & $\begin{array}{l}\text { Maternal shock without mention of trauma } \\
\text { SID }\end{array}$ & $785 \cdot 5$ & $785 \cdot 5$ \\
\hline & $\begin{array}{l}\text { Spontaneous abortion } \\
\text { Disorders related to short gestat }\end{array}$ & $761 \cdot 8$ & $761 \cdot 8$ & 9 & Sudden infant death syndrome (SIDS) & & \\
\hline & & $\begin{array}{l}765 \cdot 0 \\
769 \cdot 0\end{array}$ & $\begin{array}{l}765 \cdot 1 \\
769 \cdot 0\end{array}$ & & $\begin{array}{l}\text { Sudden infant death syndrome (SIDS) } \\
\text { Instantaneous death }\end{array}$ & $\begin{array}{l}798 \cdot 0 \\
798 \cdot 1\end{array}$ & $\begin{array}{l}798 \cdot 0 \\
798 \cdot 1\end{array}$ \\
\hline & Interstitial emphysema etc & $770 \cdot 2$ & $770 \cdot 2$ & & $\begin{array}{l}\text { Instantaneous deatn } \\
\text { Unexplained death }\end{array}$ & $\begin{array}{l}198 \cdot 1 \\
798 \cdot 2\end{array}$ & $\begin{array}{l}798 \cdot 1 \\
798 \cdot 2\end{array}$ \\
\hline & Atelectasis & $770 \cdot 4$ & $770 \cdot 5$ & & Unattended death & $798 \cdot 9$ & 798.9 \\
\hline & Respiratory problems after birth & $770 \cdot 7$ & $770 \cdot 8$ & & Respiratory failure & $799 \cdot 1$ & $799 \cdot 1$ \\
\hline & Intraventricular haemorrhage & $772 \cdot 1$ & $772 \cdot 1$ & $\mathbf{0}$ & Other conditions occurring in $1991 \mathrm{n}$ & & \\
\hline & Neonatal jaundice associated with preterm birth & $774 \cdot 2$ & $\mathbf{7 7 4 \cdot 2}$ & & Acidosis & $276 \cdot 2$ & $276 \cdot 2$ \\
\hline & Kernicterus not due isoimmunisation & $\mathbf{7 7 4} \cdot 7$ & $774 \cdot 7$ & & Volume depletion & $276 \cdot 5$ & $276 \cdot 5$ \\
\hline & Anaemia of prematurity & $776 \cdot 6$ & $776 \cdot 6$ & & Hyperpotas & 27 & $276 \cdot 7$ \\
\hline & Necrotising enterocolitis & $777 \cdot 5$ & $777 \cdot 5$ & & ecified) & $285 \cdot 9$ & $285 \cdot 9$ \\
\hline & Legal termination of pregnancy & $779 \cdot 6$ & $779 \cdot 6$ & & Encephalopathy (unspecified) & $348 \cdot 3$ & $348 \cdot 3$ \\
\hline 4 & Asphyxia, anoxia, or trauma & & & & 'Other' myoneural disorder & $358 \cdot 8$ & $358 \cdot 8$ \\
\hline & Antepartum haemorrhage (maternal codes) & $641 \cdot 0$ & $642 \cdot 9$ & & cardial infarction & $410 \cdot 0$ & $410 \cdot 0$ \\
\hline & Maternal hypertension (perinatal code) & $760 \cdot 0$ & $760 \cdot 0$ & & Primary pulmonary hypertension & $416 \cdot 0$ & $416 \cdot 0$ \\
\hline & Effects of maternal complica & $761 \cdot 6$ & $761 \cdot 7$ & & & $428 \cdot 0$ & $428 \cdot 0$ \\
\hline & Placental problems (perinatal codes) & $762 \cdot 0$ & $762 \cdot 2$ & & of the & $434 \cdot 0$ & $434 \cdot 9$ \\
\hline & Cord problems (perinatal codes) & $762 \cdot 4$ & $762 \cdot 6$ & & scular & $437 \cdot 1$ & $437 \cdot 3$ \\
\hline & Other complications of labour/delivery (perinatal code) & $763 \cdot 0$ & $763 \cdot 9$ & & Other venous embolism or thrombosis & $453 \cdot 0$ & 453.9 \\
\hline & Slow fetal growth/malnutrition (perinatal code) & $764 \cdot 0$ & $764 \cdot 9$ & & Unspecified hypotension & $458 \cdot 9$ & $458 \cdot 9$ \\
\hline & Long gestation/high birth weigh//birth trauma/hypoxia & $766 \cdot 0$ & $768 \cdot 9$ & & gestion and hypostasis & $514 \cdot 0$ & $514 \cdot 0$ \\
\hline & Massive aspiration syndrome & $770 \cdot 1$ & $770 \cdot 1$ & & Other pneumopathy & $516 \cdot 8$ & $516 \cdot 8$ \\
\hline & Subarachnoid haemorrhage & $772 \cdot 2$ & $772 \cdot 2$ & & the lung. & $518 \cdot 8$ & $518 \cdot 8$ \\
\hline & Other and unspecified postnatal respiratory problems & $779 \cdot 0$ & $779 \cdot 2$ & & Other diseases of the respiratory system & $519 \cdot 8$ & $519 \cdot 8$ \\
\hline 5 & External & & & & al syndrome & $572 \cdot 4$ & $572 \cdot 4$ \\
\hline & Malnutrition & $260 \cdot 0$ & 263.9 & & Unspecified acute renal failure & $584 \cdot 9$ & $584 \cdot 9$ \\
\hline & Pneumonitis due to solids or liquids & $507 \cdot 0$ & $507 \cdot 8$ & & Renal failure & $586 \cdot 0$ & $586 \cdot 0$ \\
\hline & Hypothermia or scleroderma & $778 \cdot 1$ & $778 \cdot 3$ & & Intestinoureteric fistula or renal infarction & 593.8 & $593 \cdot 8$ \\
\hline & Feeding problems in newborn & $779 \cdot 3$ & $779 \cdot 3$ & & Congenital deformity spine/congenital dislocatic & $754 \cdot 2$ & $\mathbf{7 5 4 \cdot 3}$ \\
\hline & Other injury or poisoning & $800 \cdot 0$ & $999 \cdot 9$ & & Patent ductus arteriosus & $747 \cdot 0$ & $\mathbf{7 4 7 \cdot 0}$ \\
\hline & & & & & Maternal renal and hypertensive disorder & $760 \cdot 1$ & $760 \cdot 1$ \\
\hline & & & & & Pulmonary haemorrhage & $770 \cdot 3$ & $770 \cdot 3$ \\
\hline & & & & & Other maternal disease or injury & $761 \cdot 3$ & $761 \cdot 5$ \\
\hline & & & & & Other and ill defined (other/unspecified) & $779 \cdot 8$ & 779.9 \\
\hline & & & & & Debility unspecified & $799 \cdot 3$ & $799 \cdot 3$ \\
\hline & & & & & Unknown & $799 \cdot 6^{\star}$ & $799 \cdot 9$ \\
\hline
\end{tabular}

The position of the mention on the certificate will indicate whether the cause is maternal or infant. ${ }^{\star}=$ OPCS code.

immaturity; and (c) to avoid confusion, causes due to oxygenation problems thought to be related to immaturity are selected out before those in other infants.

Table 1 gives the current full list of ICD codes for the conditions which constitute each category.
VALIDATION OF THE CLASSIFICATION

The validity of the algorithm was tested in several ways. First any break in trend was sought in the distribution and pattern of the groups derived from the same ICD codes before and after the use of the new certificate and the algorithm. As stated earlier, one of the 
Table 2 Neonatal deaths 1991: total mentions by place on certificate

\begin{tabular}{lcr}
\hline Place on certificate & $\begin{array}{l}\text { No }(\%) \\
\text { mentions }\end{array}$ & $\begin{array}{r}\text { Cases } \\
(\%)^{\star}\end{array}$ \\
\hline First main infant & $3041(37 \cdot 6)$ & $99 \cdot 6$ \\
Second main infant & $587(7 \cdot 2)$ & $19 \cdot 2$ \\
Third main infant & $121(1 \cdot 5)$ & $4 \cdot 0$ \\
First main maternal & $767(9 \cdot 5)$ & $25 \cdot 1$ \\
Second main maternal & $84(1 \cdot 0)$ & $2 \cdot 8$ \\
Third main maternal & $11(0 \cdot 1)$ & $0 \cdot 4$ \\
First other infant & $1985(24 \cdot 5)$ & $65 \cdot 0$ \\
Second other infant & $795(9 \cdot 8)$ & $26 \cdot 0$ \\
Third other infant & $257(3 \cdot 2)$ & $8 \cdot 4$ \\
Fourth other infant & $72(0 \cdot 9)$ & $2 \cdot 4$ \\
Fifth other infant & $21(0 \cdot 3)$ & $0 \cdot 7$ \\
Sixth other infant & $8(0 \cdot 1)$ & $0 \cdot 3$ \\
Seventh other infant & $1(0 \cdot 0)$ & $0 \cdot 0$ \\
Eighth other infant & $1(0 \cdot 0)$ & $0 \cdot 0$ \\
First other maternal & $301(3 \cdot 7)$ & $9 \cdot 9$ \\
Second other maternal & $44(0 \cdot 5)$ & $1 \cdot 4$ \\
Third other maternal & $2(0 \cdot 0)$ & $0 \cdot 1$ \\
Fourth other maternal & $0(0 \cdot 0)$ & $0 \cdot 0$ \\
Total mentions & $8098(100)$ & \\
Total cases & 3052 & \\
Mentions/case & $2 \cdot 7$ & \\
T.t. & &
\end{tabular}

*Adds up to over $100 \%$.

requirements for the new classification was that its use should cause minimal perturbation to time trends. Second, any undue perturbation was sought in the pattern of causes between the neonatal period, where the new certificate was used, and those in the postneonatal period where the old certificate was still in use. Lastly, the data were examined to see whether the use of the new classification revealed the expected relations between birth weight and cause group.

\section{Results}

\section{MENTIONS IN DIFFERENT PARTS OF THE}

NEONATAL CERTIFICATE

Before describing the testing of the new grouping a brief account will be given of the frequency and pattern of mentions and the order in which they occur on the new certificates. For simplicity, only data for neonatal deaths registered in 1991 will be described in detail.

In 1991 there were 3052 neonatal deaths registered in England and Wales. In total there were 8098 coded mentions of conditions derived from the medical certificate of death. Overall, there was an average of 2.7 mentions for each case, with $99 \%$ of the neonatal deaths having at least one mention on the main infant line, $25 \%$ at least one mention on the main maternal line, and $65 \%$ at least one mention on the other infant line. Table 2 gives the distribution of mentions. Certifying doctors often took the opportunity to enter several
Table 3 Neonatal deaths 1991: number of infants by maternal code

\begin{tabular}{lrrrrrrr}
\hline \begin{tabular}{l} 
No $\begin{array}{l}\text { Nonfant } \\
\text { codes }\end{array}$ \\
\cline { 2 - 9 }
\end{tabular} & \multicolumn{1}{l}{ No maternal codes } & & & & & \\
\hline 0 & 2 & 3 & 0 & 1 & 0 & 0 & 6 \\
1 & 710 & 116 & 54 & 8 & 0 & 1 & 889 \\
2 & 758 & 249 & 79 & 19 & 0 & 0 & 1105 \\
3 & 424 & 169 & 38 & 6 & 2 & 0 & 639 \\
4 & 175 & 67 & 23 & 3 & 2 & 0 & 270 \\
5 & 54 & 24 & 13 & 2 & 0 & 0 & 93 \\
6 & 23 & 3 & 4 & 1 & 1 & 0 & 32 \\
7 & 9 & 1 & 1 & 0 & 0 & 0 & 11 \\
8 & 1 & 2 & 1 & 0 & 0 & 0 & 4 \\
9 & 1 & 1 & 0 & 1 & 0 & 0 & 3 \\
All & 2157 & 635 & 213 & 41 & 5 & 1 & 3052
\end{tabular}

causes on each line, particularly the 'other' infant causes. Table 3 presents a tabulation of total infant mentions by total maternal mentions.

\section{CONTRIBUTION OF MENTIONS ON DIFFERENT}

LINES TO THE CAUSE GROUPS

The expert group proposed that OPCS should send an inquiry to the notifying doctor where the final cause group was determined by a cause other than one registered as main by the certifying doctor. The proportion of neonatal deaths in which such inquiries will be required., if this recommendation is followed, can be estimated by examining where on the certificate the mention which determined the final cause grouping was placed. It should be noted that in the small proportion of cases where there is clearly an illogical sequence on the certificate the coding clerks in OPCS will change the order, and the following analysis is based on the amended order.

Table 4 shows that in the 3052 deaths registered in 1991 the cause group was determined by the first, second, or third main infant mentions in $2682(88 \%), 85(3 \%)$, and six $(0.2 \%)$ respectively, and by any main maternal mention in $32(1 \%)$. Only $73 \%$ of the allocations to the congenital malformation and defect group had been based on the first main infant cause, but this cause had determined the grouping of $97 \%$ of the cases allocated to immaturity group, and $80 \%$ of those allocated to asphyxia, anoxia, and/or trauma.

In about $10 \%$ of cases use of the algorithm led to a cause based on a mention on an 'other' line. This is the situation which would prompt an inquiry to the certifying doctor to confirm that the suggested grouping is in accordance with the clinical opinion.

Table 4 Mention which determined hierarchial group

\begin{tabular}{|c|c|c|c|c|c|c|c|c|c|c|}
\hline Group & $\begin{array}{l}\text { First } \\
\text { main } \\
\text { infant }\end{array}$ & $\begin{array}{l}\text { Second } \\
\text { main } \\
\text { infant }\end{array}$ & $\begin{array}{l}\text { Third } \\
\text { main } \\
\text { infant }\end{array}$ & $\begin{array}{l}\text { First } \\
\text { main } \\
\text { maternal }\end{array}$ & $\begin{array}{l}\text { Second } \\
\text { main } \\
\text { maternal }\end{array}$ & $\begin{array}{l}\text { First } \\
\text { other } \\
\text { infant }\end{array}$ & $\begin{array}{l}\text { Second } \\
\text { other } \\
\text { infant }\end{array}$ & $\begin{array}{l}\text { Third } \\
\text { other } \\
\text { infant }\end{array}$ & $\begin{array}{l}\text { Fourth } \\
\text { other } \\
\text { infant }\end{array}$ & Total \\
\hline Congenital malformation & 710 & 30 & 4 & 0 & 0 & 175 & 43 & 10 & 1 & 973 \\
\hline Antepartum infection & 11 & 4 & 0 & 0 & 0 & 11 & 3 & 0 & 0 & 29 \\
\hline Immaturity & 1339 & 43 & 2 & 0 & 0 & 0 & 0 & 0 & 0 & 1384 \\
\hline Asphyxia & 295 & 7 & 0 & 29 & 1 & 24 & 7 & 5 & 2 & 370 \\
\hline External factor & 20 & 0 & 0 & 0 & 0 & 0 & 0 & 0 & 0 & 20 \\
\hline Infection & 102 & 0 & 0 & 0 & 0 & 9 & 2 & 1 & 0 & 114 \\
\hline Other specified factor & 39 & 1 & 0 & 0 & 0 & 6 & 1 & 0 & 0 & 47 \\
\hline SIDS & 63 & 0 & 0 & 0 & 0 & 0 & 0 & 0 & 0 & 63 \\
\hline Other & 49 & 0 & 0 & 3 & 0 & 0 & 0 & 0 & 0 & 52 \\
\hline Total & 2682 & 85 & 6 & 32 & 1 & 225 & 56 & 16 & 3 & 3052 \\
\hline
\end{tabular}




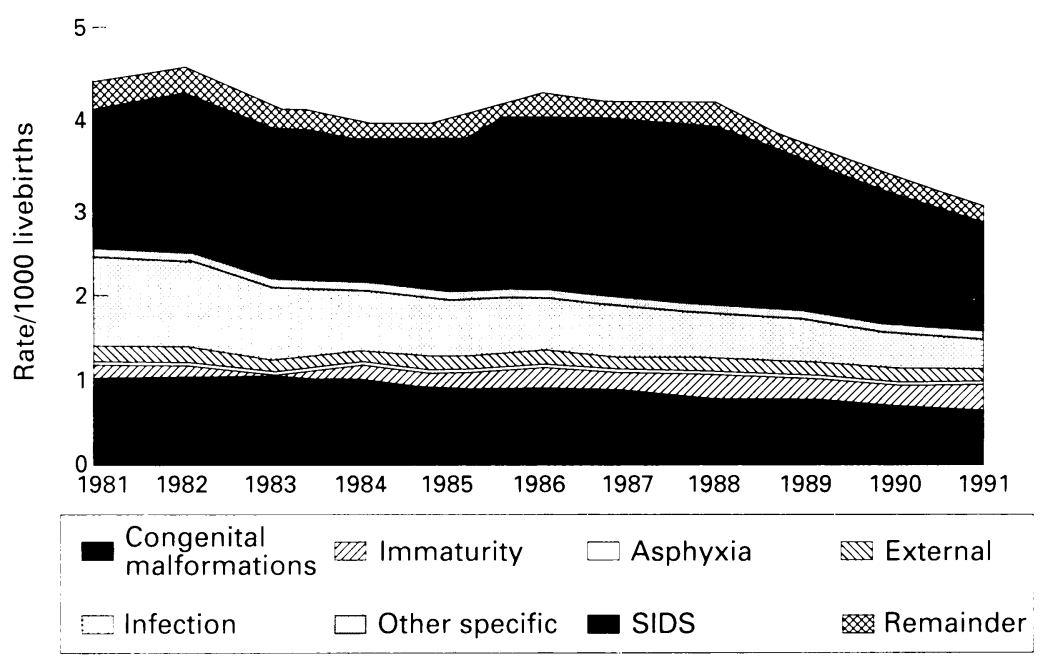

No certificate change

Figure 2 Trend in cause groups of postneonatal deaths in England and Wales, 1981-91.

USING THE NEW GROUPING TO EXAMINE THE EFFECTS OF THE NEW CERTIFICATE ON TRENDS OVER TIME

To examine the effect on trends, rates using the same ICD grouping were plotted based on underlying cause from 1981 to 1985 , and the grouping derived from all mentioned conditions using the algorithm from 1986 to 1991 . Figure 2 shows that the overall trends for postneonatal deaths, where there has been no change in certification, remain reasonably consistent over the two time periods, suggesting that the classification is generally robust. Figure 3 for the neonatal deaths, however, shows that in the 1986 data there were small stepwise increases in the congenital malformation and the immaturity groups. These are attributable to the increases in the numbers so classified using all the mentions on the new certificate. After 1986 the trends are consistent.

USING THE NEW GROUPING TO EXAMINE TRENDS BY AGE AT DEATH

Crude trends of infant deaths obscure the fact that almost half of all neonatal deaths occur in

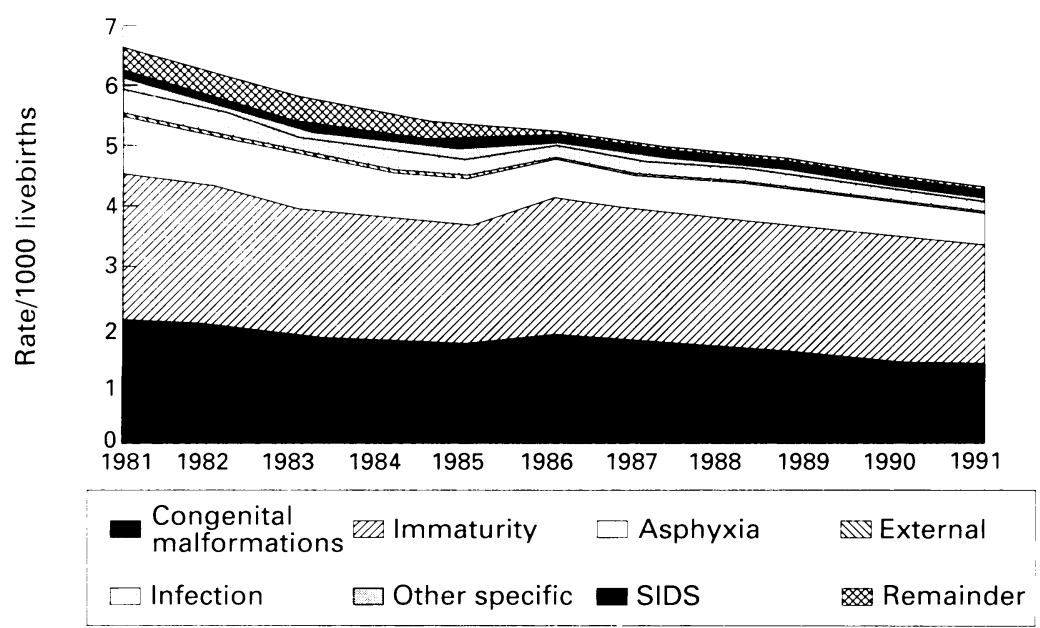

Change of certificate 1986

Figure 3 Trends in cause groups of neonatal deaths in England and Wales, 1981-91. the first week of life, most in the first day. Figure 4 gives the new cause groups by age subgroups for infant deaths registered in 1991 . The overwhelming numerical importance of immaturity as a cause in the deaths at early ages is clearly shown. Figure 5 gives the same data but expressed as the percentage due to different causes groups at different ages. Congenital malformations and defects are consistently important, though more so during the first three weeks of life, whereas the predominance of immaturity in the first weeks gives way first to postnatal infection in the fourth week, and then to sudden infant death in the second month.

It is likely that the changeover from the neonatal to the standard death certificate after four weeks of life will have affected these proportions to some extent. Nevertheless, the expected increase in sudden infant deaths from the second week to the third month shows a fairly steady trend. Similarly, there is a consistent decrease in deaths due to congenital malformations or defects, crossing over the ages at which there is a change in the type of certificate used. The proportion of other deaths whose causes did not fall into one of the defined groups increased fairly sharply after the neonatal period, however (fig 5). This probably reflects the restricted amount of information available from the single underlying cause derived from the standard death certificate.

USING BIRTH WEIGHT DATA TO VALIDATE THE NEW GROUPS

As birth weight is currently not available for infant deaths until after the annual linkage with birth registration data, the new classification does not initially take birth weight into account. An important method of validation is therefore to study the birth weight distributions of the cause groups, particularly the immaturity group which would be expected to comprise mostly infants of very low birth weight.

In 1986 less than $1 \%$ of all live births and only $3 \%$ of neonatal deaths did not have a recorded birth weight, although this increased to $5 \%$ for deaths in the other cause group and $6 \%$ in that due to external causes (which include unattended labour) (table 5). Eighty nine per cent of the 'immaturity' group of neonatal deaths were known to have weighed less than $2000 \mathrm{~g}$ and $5 \%$ less than $500 \mathrm{~g}$. In 1991 levels of unstated birth weight were four times as common in neonatal deaths as in live births, but even so $79 \%$ of the immaturity group were known to have weighed less than $2000 \mathrm{~g}$ and $8 \%$ less than $500 \mathrm{~g}$, whereas $17 \%$ were of unknown weight. Gestational age, which would be important in this context, is not recorded at the registration of live births.

The grouping used showed how misleading it may be to classify deaths as due to immaturity by birth weight alone. In the years where birth weight is most complete, $1986-8,25 \%$ of the neonatal deaths classified as due to congenital defects, $64 \%$ of the small group of those with 
congenital infections, and $45 \%$ of those of other specific causes weighed less than $2000 \mathrm{~g}$. In these years only $2 \%$ of all live births were of this birth weight group. Only in the group including causes of birth asphyxia and trauma is there a predominance of infants of heavier weight, $59 \%$ weighing $2500 \mathrm{~g}$ or more and $20 \%$ weighing $3500 \mathrm{~g}$ or more, though this proportion is relatively low compared with the $37 \%$ of live births of this weight in the same period.

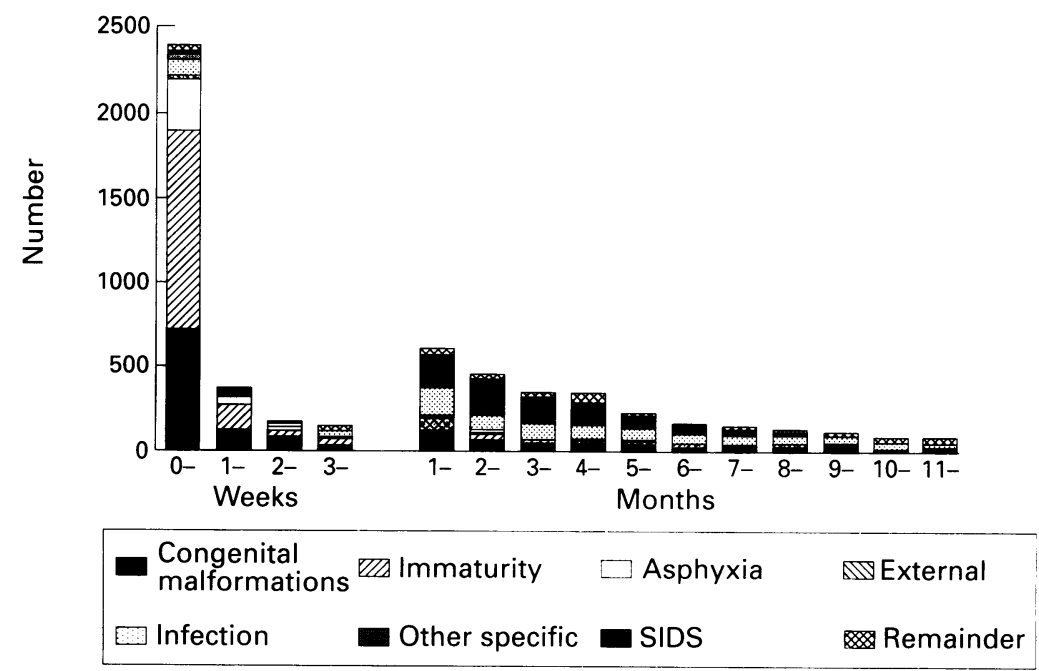

Figure 4 Trends in numbers in causes of infant deaths by age subgroups in England and Wales, 1991.

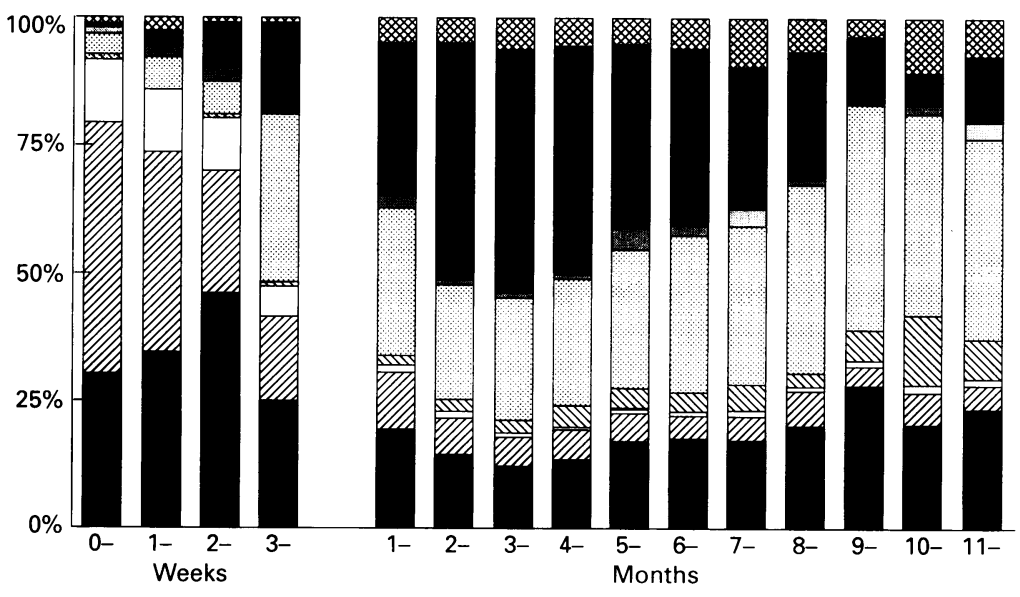

$\begin{aligned} & \text { Congenital } \\ & \text { malformations }\end{aligned}$
$\square$ Infection Immaturity $\square$ Asphyxia
Other specific $\square$ SIDS

Figure 5 Trends in percentage of causes of infant deaths by age subgroups in England and Wales, 1991.
CONTRIBUTION OF MATERNAL CAUSES

For neonatal deaths the maternal causes were more valuable for looking at associations between mentions than for determining the cause classification. Maternal causes were most often given in the asphyxia group, where $15 \%$ of all mentions fell into the first main maternal category. Thirty eight per cent of these referred to an antepartum haemorrhage, $9 \%$ to hypertension, and $5 \%$ to malpresentation. In only $6 \%$ was the mention that determined the cause group found on a maternal line. In the immaturity group $11 \%$ of all mentions were found in the first main maternal line, and of those $18 \%$ referred to antepartum haemorrhage, $20 \%$ to hypertension, $18 \%$ to early onset of delivery, $9 \%$ to multiple pregnancy, and $14 \%$ to problems with the amniotic cavity or membranes.

\section{Discussion}

The main aim of this paper is to describe the new OPCS hierarchical classification of causes of neonatal deaths, developed after the introduction of the new medical certificate for neonatal death which invites mentions of multiple causes. The use of this new classification has reduced the proportion of cases documented on the new form which were difficult to allocate into a single main cause group. The availability of multiple causes and the use of the algorithm slightly increased the proportion attributed to congenital malformation or defect and immaturity compared with the same grouping based on the single underlying cause derived from the old certificates. Overall, its use has caused little disturbance in terms of the continuity of trends, and the grouping had validity in terms of patterns of birthweight distribution for the different causes.

The classification is designed to help plan preventative action and to indicate the developmental period where the first insult had an effect. Of the 3052 neonatal deaths that occurred in 1991, 33\% were attributable to a congenital malformation, defect or infection and another $45 \%$ to immaturity, accounting together for $78 \%$ of the deaths. Neonatal deaths thought to be due to asphyxia, anoxia, or trauma accounted for $12 \%$ and another $4 \%$ were attributed to infections. Comparable figures for the postneonatal deaths suggested that $23 \%$ were attributable to congenital malformations or defects, $3 \%$ to immaturity, less

Table 5 Neonatal (NND) and postneonatal (PND) deaths 1986: percentage by birth weight and cause group

\begin{tabular}{|c|c|c|c|c|c|c|c|c|c|c|c|c|c|c|c|c|c|c|c|c|}
\hline \multirow{2}{*}{$\begin{array}{l}\text { Birth weight } \\
\text { (g) }\end{array}$} & \multicolumn{2}{|l|}{ Total } & \multicolumn{2}{|c|}{$\begin{array}{l}\text { Congenital } \\
\text { malformation }\end{array}$} & \multicolumn{2}{|c|}{$\begin{array}{l}\text { Antepartum } \\
\text { infection }\end{array}$} & \multicolumn{2}{|c|}{ Immaturity } & \multicolumn{2}{|c|}{$\begin{array}{l}\text { Asphyxia, } \\
\text { anoxial } \\
\text { trauma }\end{array}$} & \multicolumn{2}{|c|}{ External } & \multicolumn{2}{|c|}{ Infection } & \multicolumn{2}{|l|}{$\begin{array}{l}\text { Other } \\
\text { specific }\end{array}$} & \multicolumn{2}{|l|}{ SIDS } & \multicolumn{2}{|l|}{ Other } \\
\hline & $N N D$ & $P N D$ & $N N D$ & $P N D$ & $N N D$ & $P N D$ & $N N D$ & $P N D$ & $N N D$ & $P N D$ & $N N D$ & $P N D$ & $N N D$ & $P N D$ & $N N D$ & $P N D$ & $N N D$ & $P N D$ & $N N D$ & $P N D$ \\
\hline$\leqslant 500$ & $2 \cdot 3$ & $0 \cdot 1$ & $0 \cdot 4$ & 0.0 & $0 \cdot 0$ & $0 \cdot 0$ & $4 \cdot 9$ & 1.9 & 0.5 & $0 \cdot 0$ & $0 \cdot 0$ & $0 \cdot 0$ & $0 \cdot 0$ & 0.0 & 0.0 & 0.0 & 0.0 & 0.0 & $0 \cdot 0$ & $0 \cdot 0$ \\
\hline 501-999 & $28 \cdot 5$ & $4 \cdot 3$ & $5 \cdot 7$ & $0 \cdot 7$ & $27 \cdot 3$ & $0 \cdot 0$ & $56 \cdot 2$ & $51 \cdot 2$ & $11 \cdot 0$ & $6 \cdot 9$ & $6 \cdot 3$ & $3 \cdot 5$ & $11 \cdot 7$ & 1.9 & $13 \cdot 3$ & $3 \cdot 9$ & $0 \cdot 0$ & 0.9 & $25 \cdot 0$ & $3 \cdot 8$ \\
\hline $1000-1499$ & $15 \cdot 5$ & $4 \cdot 4$ & $12 \cdot 2$ & $2 \cdot 7$ & $22 \cdot 7$ & $0 \cdot 0$ & $21 \cdot 0$ & $32 \cdot 5$ & $6 \cdot 7$ & $3 \cdot 5$ & $6 \cdot 3$ & 3.5 & $20 \cdot 7$ & $2 \cdot 4$ & $15 \cdot 6$ & $3 \cdot 9$ & 1.5 & $2 \cdot 5$ & 12.5 & $4 \cdot 5$ \\
\hline $1500-1999$ & $10 \cdot 4$ & $6 \cdot 7$ & $15 \cdot 3$ & $9 \cdot 6$ & $13 \cdot 6$ & $0 \cdot 0$ & $7 \cdot 3$ & $6 \cdot 3$ & $6 \cdot 9$ & 0.0 & $0 \cdot 0$ & $2 \cdot 4$ & $8 \cdot 1$ & $7 \cdot 1$ & $15 \cdot 6$ & $9 \cdot 8$ & $2 \cdot 9$ & $5 \cdot 6$ & $17 \cdot 5$ & $8 \cdot 3$ \\
\hline $2000-2499$ & $9 \cdot 6$ & $10 \cdot 0$ & $16 \cdot 8$ & $17 \cdot 4$ & $4 \cdot 5$ & 0.0 & $2 \cdot 0$ & 1.3 & $12 \cdot 4$ & $3 \cdot 5$ & $18 \cdot 8$ & $10 \cdot 6$ & $18 \cdot 0$ & $11 \cdot 4$ & $15 \cdot 6$ & $11 \cdot 8$ & $5 \cdot 9$ & $7 \cdot 4$ & $5 \cdot 0$ & $10 \cdot 8$ \\
\hline $2500-2999$ & $10 \cdot 6$ & $21 \cdot 4$ & 18.8 & $24 \cdot 3$ & $18 \cdot 2$ & $50 \cdot 0$ & $2 \cdot 0$ & 1.9 & 13.6 & $17 \cdot 2$ & 12.5 & $21 \cdot 2$ & $11 \cdot 7$ & $20 \cdot 5$ & $17 \cdot 8$ & $17 \cdot 7$ & $17 \cdot 6$ & $22 \cdot 8$ & $7 \cdot 5$ & $23 \cdot 6$ \\
\hline $3000-3499$ & $11 \cdot 7$ & $29 \cdot 6$ & $16 \cdot 8$ & $26 \cdot 1$ & 0.0 & $50 \cdot 0$ & 1.6 & $1 \cdot 3$ & $25 \cdot 1$ & $37 \cdot 9$ & $31 \cdot 3$ & $35 \cdot 3$ & $18 \cdot 9$ & $31 \cdot 7$ & 8.9 & $27 \cdot 5$ & $36 \cdot 8$ & 33.9 & $22 \cdot 5$ & $24 \cdot 2$ \\
\hline$\geqslant 3500$ & $8 \cdot 6$ & $23 \cdot 0$ & $11 \cdot 4$ & $18 \cdot 7$ & $9 \cdot 1$ & $0 \cdot 0$ & $1 \cdot 6$ & 0.6 & $20 \cdot 1$ & $27 \cdot 6$ & $18 \cdot 8$ & 23.5 & $9 \cdot 9$ & $24 \cdot 8$ & $11 \cdot 1$ & $23 \cdot 5$ & $35 \cdot 3$ & $26 \cdot 9$ & $5 \cdot 0$ & $24 \cdot 2$ \\
\hline Not stated & $3 \cdot 0$ & $0 \cdot 4$ & $2 \cdot 6$ & $0 \cdot 4$ & $4 \cdot 5$ & $0 \cdot 0$ & $3 \cdot 3$ & $3 \cdot 1$ & $3 \cdot 6$ & 3.5 & $6 \cdot 3$ & $0 \cdot 0$ & 0.9 & $0 \cdot 2$ & $2 \cdot 2$ & $2 \cdot 0$ & $0 \cdot 0$ & $0 \cdot 0$ & $5 \cdot 0$ & 0.6 \\
\hline Total $(100 \%)$ & 3449 & 2760 & 1253 & 551 & 22 & 4 & 1476 & 160 & 418 & 29 & 16 & 85 & 111 & 420 & 45 & 51 & 68 & 1303 & 40 & 157 \\
\hline
\end{tabular}


than $1 \%$ to asphyxia or trauma, $23 \%$ to infections, and $36 \%$ to sudden infant death. The data confirm what is now well recognised, namely that most of the neonatal deaths occur because of prenatal problems, contrasting with the postneonatal deaths which are more likely to be due to environmental hazards.

A simplified form of this classification is being used for the confidential inquiry into stillbirths and infant deaths currently under way in England and Wales. ${ }^{16}$ The analysis of these data will give further insight into its usefulness, but the first year's full data will not be available until 1994.

The new grouping will enable us again to compare the associations of social class, region of residence, maternal age, and marital status with similar causes in neonatal and postneonatal deaths and has allowed the use of all the causes on the neonatal certificate. Work is in preparation to allow similar data from stillbirth certificates to be analysed in the same way.

The advice and help of the members of the Expert Advisory Group (Dr John Ashley, Dr John Fox, Dr Edmund Hey, D Stephen Leadbetter, Professor Malcolm Levene, Dr Jean Keeling, Ms Alison Macfarlane, Dr Michael Maresh, and Dr Jo Walsworth-Bell) is gratefully acknowledged.

1 Baird D, Walker J, Thomson AM. The causes and prevention of stillbirths and first week deaths. Part III. A classification of deaths by clinical causes: the effect of age, parity and length of gestation on death rates by cause. fournal of Obstetrics and Gynaecology of the British Empire 1954; 61: 433-48.

2 Bound JP, Butler NR, Spector WG. Classification and causes of perinatal mortality. BMF 1956; ii: 1191-6.

3 Bound JP, Butler NR, Spector WC. Classification and causes of perinatal mortality. BMF 1956; ii: $1260-5$.

4 Baird D, Thomson AM. The survey perinatal deaths reclassified by special clinico-pathological assessment. In: Butler NR, Alberman ED, eds. Perinatal problems. The second report of the 1958 British Perinatal Mortality Survey. Edinburgh: Churchill Livingstone, 1969; 200-10.

5 Wigglesworth JS. Monitoring perinatal mortality - a pathophysiological approach. Lancet 1980; ii: 684-6.

6 Cole SK, Hey EN, Thomson AM. Classifying perinatal death: an obstetric approach. Br $\mathcal{F}$ Obstet Gynaecol 1986; 93: 1204-12.

7 Hey EN, Lloyd DJ, Wigglesworth JS. Classifying perinatal death: fetal and neonatal factors. Br $\mathcal{F}$ Obstet Gynaecol 1986; 93: 1213-23.

8 Barron SL. How can we improve perinatal surveillance. $\mathrm{Br} \mathcal{f}$ Obstet Gynaecol 1986; 93: 1201-3.

9 World Health Organisation. International classification of diseases. 9th Revision. Geneva: WHO, 1977: 731-3.

10 House of Commons Select Committee on Health and Social Services. Perinatal and neonatal mortality. London HMSO, 1980. (Cmnd 663-1).

11 Murphy M, Botting B. Contrasts in the multiple causes of stillbirth, neonatal death and postneonatal death. $\mathfrak{f}$ Epidemiol Community Health 1989; 43: 343-5.

12 Gedalla B, Alderson MR. Pilot study of revised stillbirth and neonatal death certificates. Arch Dis Child 1984; 59 976-82.

13 Office of Population Censuses and Surveys. Mortality statistics childhood. Annual reference volume series DH6. London: HMSO, 1986-91.

14 Office of Population Censuses and Surveys. Mortality statistics perinatal and infant; social and biological factors. Annual reference volume series DH3. London HMSO, Annual refer

15 Cole S, Hartford RB, Bergsio P, McCarthy B. International collaborative effort (ICE) on birthweight, plurality, perinatal, and infant mortality. III. A method of grouping underlying causes of infant death to aid international comparisons. Acta Obstet Gynecol Scand 1989; 68: 113-7.

16 National Advisory Body. The confidential enquiry into stillbirths and deaths in infancy. Report March 1992-July 1993. 